\title{
A highly efficient prediction of delamination migration in laminated composites using the extended cohesive damage model
}

\author{
$\mathrm{X} \mathrm{Li}$ and $\mathrm{J}$ Chen* \\ School of Civil Engineering and Surveying, University of Portsmouth \\ Portland Street, Portsmouth PO1 3AH, UK
}

\begin{abstract}
The extended cohesive damage model (ECDM) has been recently developed by authors for predicting multicrack failure mechanism in fibre materials. The ECDM is Partition of Unity Method (PUM) based and is a condensed FEM formulation through eliminating the enriched degree of freedoms (DoFs) for nonlinear fracture analysis. To account for the cohesive crack effect, an equivalent damage scalar relating to strain filed is introduced in terms of energy dissipation during the post-failure process to characterize the damage evolution. The ECDM is capable of characterizing discontinuity with conventional DoFs only, thus it is significantly efficient in modelling multicrack propagation. This paper investigates the performance of the ECDM through single element tests as well as modelling the delamination migration in laminated composites. Detailed comparison with experimental work and standard cohesive zone model $(\mathrm{CZM})$ is carried out. This investigation shows the ECDM exhibits excellent efficiency and accuracy compered to CZM. The ECDM can reduce the CPU time in prediction of delamination migration of a laminated composite specimen by more than $90 \%$ compared to CZM. Therefore, the ECDM is a robust computational approach for highly efficient predicting delamnation migration in laminated composites.
\end{abstract}

Keywords: ECDM, delamination migration, computing efficiency, multicrack propagation

\footnotetext{
* Corresponding author.

E-mail address: jiye.chen@port.ac.uk (J. Chen).
} 


\section{Introduction}

The solution of classical FEM essentially depends on approximation properties of polynomial shape functions. Hence its application generally requires that the solutions possess smooth features so as to obtain optimal accuracy [1]. Nevertheless, material fracture may lead to nonsmooth characteristics of material behavior, such as high gradients/singularities in stress and strain fields, strong discontinuities in the displacement field as in case of cracked material mediums. In such scenario, the FEM becomes computationally expensive, and be hardly converge to a physical solution. Therefore, the classical FEM will not work well anymore when dealing with the computational material failure consisting of high strain gradient and material nonlinearity. A number of instances can be found regarding the nonlinear material modelling, in which the standard FEM performs struggling as an inefficient method [2]. To not only predict the structural strength, i.e. failure load, but also the post-failure course correctly, FEM based computational algorithms, which are capable of dealing with the governing equations with high nonlinearity, and are satisfactory in computational robustness and stability, accuracy, efficiency, are still greatly required in engineering fields.

In heterogeneous materials, local stress and strain field can significantly varies due to local material features, which poses special challenges to computational modelling. Moreover, the heterogeneity usually brings more complex failure mechanisms [3-5]. Fracture can be found during damage evolution not only on the material demarcation but also on other surfaces that cannot be specified a priori. Sometimes, multiple failure mechanisms happen simultaneously and multiple failures coupling during damage evolution makes prediction of multiple failure behavior a real challenge in heterogeneous materials [5]. Normally, the failure characteristic of heterogeneous materials is at the same scalar with the configuration of heterogeneity. Therefore, the continuum damage strategy based on the homogeneity assumption for simulation [6] always performs struggling when encountering material heterogeneity, e.g., Fiber Reinforced Polymer (FRP) composites.

Within the Finite Element Method framework, the commonly accepted approaches in modelling FRP fracture are the Virtual Crack Closure Technique (VCCT) [7, 8] and Cohesive Zone Method (CZM) (e.g. [9, 10]). Nevertheless, both VCCT and CZM assume that Fractures, 
e.g., delamination, grow along an interface between elements. It is required that the fracture surfaces coincide with the element boundary when applying VCCT and CZM in dealing with non-continuous problems, e.g. crack simulation [9]. With the complexity of material configuration and failure mechanisms of FRP composites, this limitation manifests itself more severely. Consequently using conventional FEM on the crack propagation analysis, the crack configuration must be generated in a priori to ensure that the element boundary and discontinues surfaces coincide.

In order to solve the above mentioned problems in discontinuous analysis using the conventional finite element method, some scholars have done considerable efforts, and provided some corresponding solutions. Babuska and Melenk [11] proposed the Partition of Unity Method (PUM), in the implementation of which, no mesh regeneration is required in the characterization of discontinuity growth and the finite element boundary would no longer need to be the discontinuity surface. The essential development of FEM method focuses on the extension and improvement of the variational principle and the approximation space. For a long time, the progress and development of the finite element method is closely connected with the expansion and development of the basic approximation space. In the past decades, in the framework of PUM, a rapid development has been made to conduct the problems containing physical discontinuities within continuum solids (12-16). Among all these PUM based approaches, the Generalized Finite Element Method (GFEM), Embedded Finite Element Method (EFEM) and eXtended Finite Element Method (XFEM) are three main expressions in the class of so called discrete damage approach (DDA), which are developed based on the combination of the classical Finite Element Method (FEM) and the PUM.

The major drawback of the above PUM related methods is the enrichment terms because additional DoFs or nodes certainly bring expensive computational cost. Sometimes, it tends to be even impossible to achieve convergent solution when encountering highly strong nonlinearity [16]. In consideration of this drawback, a novel Extended Cohesive Damage Model (ECDM) was recently developed by authors' previous work to describe the multicrack propagation in fibre composites [23, 24]. The advantages of the ECDM in comparison with 
other parallel damage modelling approaches are: 1) permitting modelling crack propagation without the dependence of mesh topology; 2) offering greatly improved numerical accuracy, efficiency and robustness; 3) driving the damage factor based on the thermal dissipation enables the model conforms with thermodynamic consistency. In addition, it demonstrates that the derived ECDM formulations can be easily implemented into any standard FE package, including commercial software package as an add-in to the element library. The features of the ECDM enable it a prospect tool to efficiently characterize arbitrary material heterogeneity and the progressive damage evolution along material interfaces, and to account for coupling of multiple failure mechanisms in a physically consistent manner.

\section{The ECDM formulas of a 2D 4-node quadrilateral element}

The theoretical derivation of ECDM in dealing with 2D cohesive crack problem has been completed in authors' previous work [23, 24]. Herein, the basic formulas of the ECDM implemented as a 2D 4-node element is conducted. In Fig. 1, a 2D quadrilateral 4-node element is presented in a physical coordinate system, and the corresponding element is presented in a natural coordinate system.

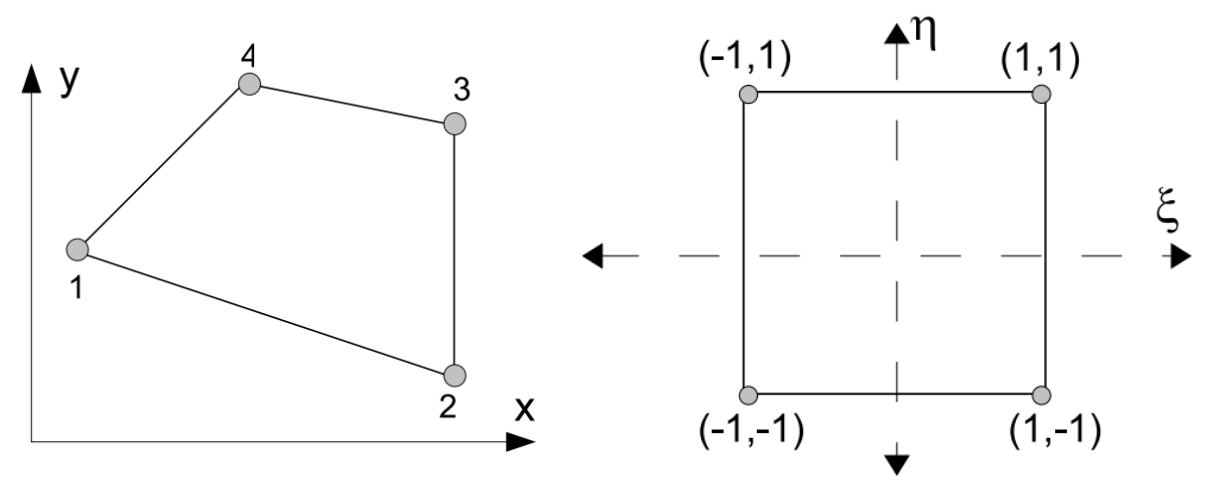

Fig. 1. (a) The ECDM quadrilateral element presented in a physical coordinate $(x, y)$ system; (b) the mapping element presented in a natural coordinate $(\zeta, \eta)$ system.

As the classical finite element shape functions $N_{i}(i=1,2,3,4)$ of 2D 4-node quadrilateral element satisfy the partition of unity condition, thus they could be used as partition of unity 
functions for enrichments [14]. Therefore, the displacement approximation within a specified cracked element can be expressed by Eq. (1):

$$
\left\{\begin{array}{l}
u \\
v
\end{array}\right\}=\sum_{i=1}^{4} N_{i}(\xi, \eta)\left\{\begin{array}{l}
u_{i} \\
v_{i}
\end{array}\right\}+\sum_{i=1}^{4} \operatorname{step}_{i}(\xi, \eta) N_{i}(\xi, \eta)\left\{\begin{array}{l}
u_{i}^{\prime} \\
v_{i}^{\prime}
\end{array}\right\}
$$

In which $u$ and $v$ are the displacement along $x$ axis and $y$ axis, respectively. The step function can be written as:

$$
\operatorname{step}_{i}(\xi, \eta)=\mathscr{A}_{\Gamma_{d}}(\xi, \eta)-\mathcal{H}_{\Gamma_{d}}\left(\xi, \eta_{i}\right)
$$

In the 2D quadrilateral element based on the formulations of the ECDM, the establishment of shape function matrices $\mathbf{N}$ and the discretized gradient operator matrix $\mathbf{B}$ is straightforward and in the same form with XFEM [16]. That is, $\mathbf{N}$ and $\mathbf{B}$ matrices are constructed with the standard FEM part and an enriched part.

$[\mathbf{N}]=\left[\mathbf{N}_{\mathrm{STD}}, \mathbf{N}_{\mathrm{ENR}}\right]$

In which

$$
\begin{aligned}
\mathbf{N}_{\mathrm{STD}} & =\left[\begin{array}{cccccccc}
N_{1} & 0 & N_{2} & 0 & N_{3} & 0 & N_{4} & 0 \\
0 & N_{1} & 0 & N_{2} & 0 & N_{3} & 0 & N_{4}
\end{array}\right] \\
\mathbf{N}_{\mathrm{ENR}} & =\left[\begin{array}{ccccccccc}
\text { step }_{1} N_{1} & 0 & \text { step }_{2} N_{2} & 0 & \text { step }_{3} N_{3} & 0 & \text { step }_{4} N_{4} & 0 \\
0 & \text { step }_{1} N_{1} & 0 & \text { step }_{2} N_{2} & 0 & \text { step }_{3} N_{3} & 0 & \text { step }_{4} N_{4}
\end{array}\right]
\end{aligned}
$$

The gradient matrices $\mathbf{B}$ in the implementation of the ECDM based 2D quadrilateral element can be given as:

$[\mathbf{B}]=\left[\mathbf{B}_{\mathrm{STD}}, \mathbf{B}_{\mathrm{ENR}}\right]$

In which

$$
\mathbf{B}_{\mathrm{STD}}=\left[\begin{array}{llll}
\mathrm{B}_{\mathrm{STD}}^{1} & \mathrm{~B}_{\mathrm{STD}}^{2} & \mathrm{~B}_{\mathrm{STD}}^{3} & \mathrm{~B}_{\mathrm{STD}}^{4}
\end{array}\right]
$$

and

$$
\mathbf{B}_{\mathrm{ENR}}=\left[\begin{array}{llll}
\mathrm{B}_{\mathrm{ENR}}^{1} & \mathrm{~B}_{\mathrm{ENR}}^{2} & \mathrm{~B}_{\mathrm{ENR}}^{3} & \mathrm{~B}_{\mathrm{ENR}}^{4}
\end{array}\right]
$$

In $\mathbf{B}$ matrices, $\mathrm{B}_{\mathrm{STD}}^{i}$ and $\mathrm{B}_{\mathrm{ENR}}^{i}$ are given by Eqs. (8) and (9), respectively. 


$$
\begin{aligned}
\mathrm{B}_{\mathrm{STD}}^{i} & =\left[\begin{array}{cc}
N_{i, x} & 0 \\
0 & N_{i, \mathrm{y}} \\
N_{i, \mathrm{y}} & N_{i, x}
\end{array}\right] \\
\mathrm{B}_{\mathrm{ENR}}^{i} & =\left[\begin{array}{cc}
\text { step }_{i} N_{i, x}+\text { step }_{i, x} N_{i} & 0 \\
0 & \text { step }_{i} N_{i, \mathrm{y}}+\text { step }_{i, \mathrm{y}} N_{i} \\
\text { step }_{i} N_{i, \mathrm{y}}+\text { step }_{i, \mathrm{y}} N_{i} & \text { step }_{i} N_{i, x}+\text { step }_{i, x} N_{i}
\end{array}\right]
\end{aligned}
$$

The computation of numerical integration is completed in the natural coordinate. Therefore, it is necessary to transform all computations in terms of natural coordinate. The relation regarding derivatives between the natural coordinates and physical coordinates (global coordinate) is [1]:

$$
\begin{aligned}
& \frac{\partial N_{i}}{\partial x}=\frac{\partial N_{i}}{\partial \xi} \frac{\partial \xi}{\partial x}+\frac{\partial N_{i}}{\partial \eta} \frac{\partial \eta}{\partial x} \\
& \frac{\partial N_{i}}{\partial y}=\frac{\partial N_{i}}{\partial \xi} \frac{\partial \xi}{\partial y}+\frac{\partial N_{i}}{\partial \eta} \frac{\partial \eta}{\partial y}
\end{aligned}
$$

which can be written as

$$
\left[\frac{\partial N_{i}}{\partial x}, \frac{\partial N_{i}}{\partial y}\right]=\left[\frac{\partial N_{i}}{\partial \xi}, \frac{\partial N_{i}}{\partial \eta}\right] \mathbf{J}^{-1}
$$

The matrix $\mathbf{J}$ is known as the Jacobian matrix. The Jacobian matrix of the current configuration within the $(\xi, \eta)$ natural coordinates is then given as

$$
\mathbf{J}^{-1}=\left[\begin{array}{ll}
\frac{\partial \xi}{\partial x} & \frac{\partial \xi}{\partial y} \\
\frac{\partial \eta}{\partial x} & \frac{\partial \eta}{\partial y}
\end{array}\right]
$$

With which the gradient matrices $\mathbf{B}$ in a global coordinate can be calculated using Eqs. (8) to (11). To guarantee the inevitability of the Jacobian matrices $\mathbf{J}$, det $|\mathbf{J}|$ is required to be greater than 0 , so that the $2 \mathrm{D}$ element is supposed to be a geometrically convex quadrilateral.

Fig. 2(a) presents a cracked element, which is supposed to be modelled with the ECDM. The elemental domain $\Omega_{\mathrm{e}}$ is cut by the crack into two quadrilateral domains. To construct the elemental stiffness matrix, we need to recognize the different domains as $\Omega_{e}^{+}$and $\Omega_{e}^{-}$in this element, as shown in Fig. 2(a).

Using the weak form of equilibrium equation from Bubnov-Galerkin method, the discrete form of equilibrium equation for static analysis can be written as shown in Eq. (13). 


$$
\left[\begin{array}{ll}
\mathbf{K}^{u u} & \mathbf{K}^{u a} \\
\mathbf{K}^{a u} & \mathbf{K}^{a a}
\end{array}\right]\left[\begin{array}{l}
\mathbf{u} \\
\mathbf{a}
\end{array}\right]=\left[\begin{array}{l}
\mathbf{f}_{\text {ext }}^{u} \\
\mathbf{f}_{\text {ext }}^{a}
\end{array}\right]
$$
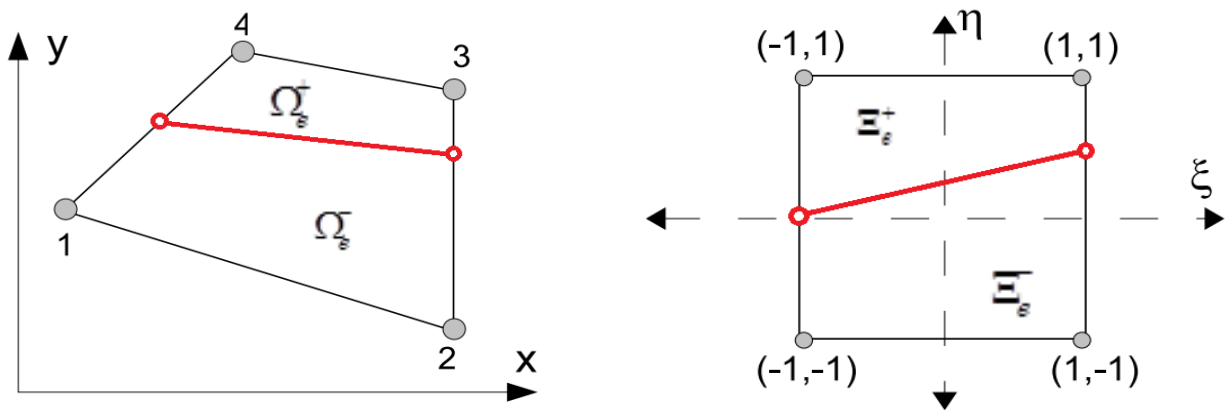

Fig. 2. The elemental configuration with a crack in (a) physical coordinate and (b) natural coordinate.

Where, $\mathbf{K}^{u u}$ and $\mathbf{K}^{a a}$ are the stiffness matrices associated with the standard FE approximation and the enriched approximation, respectively; $\mathbf{K}^{u a}$ and $\mathbf{K}^{a u}$ account for the coupling between the standard FE approximation and the enriched approximation; $\mathbf{f}_{e x t}^{u}$ and $\mathbf{f}_{\text {ext }}^{a}$ are the equivalent nodal force vectors for standard FEM DoFs and enriched DoFs, respectively; u denotes the standard DoFs while a denotes the enriched DoFs.

In Eq. (13), the equivalent nodal force vectors can be expressed as:

$$
\begin{aligned}
& \mathbf{f}_{\text {ext }}^{u}=\int_{\Gamma^{h}} \mathbf{N}_{\mathrm{STD}}{ }^{T} \overline{\mathbf{t}} d \Gamma \\
& \mathbf{f}_{\text {ext }}^{a}=\int_{\Gamma^{h}} \mathbf{N}_{\mathrm{STD}}{ }^{T}\left(\mathrm{H}_{\Gamma_{d}}(x, y)-\mathrm{H}_{\Gamma_{d}}\left(x_{i}, y_{i}\right)\right) \overline{\mathbf{t}} d \Gamma+\mathbf{f}_{c o h}
\end{aligned}
$$

where, $\Gamma_{h}$ is the boundary on which the external traction $\overline{\mathbf{t}}$ is applied. Because of the existence of the cohesive segment in the element, the internal nodal force vector due to cohesive traction $\mathbf{t}$ on the crack surface $\Gamma_{d}$ can be expressed as:

$$
\begin{aligned}
\mathbf{f}_{c o h} & =\int_{\Gamma_{d^{+}}}\left(\mathscr{H}_{\Gamma_{d}}(x, y)-\mathscr{H}_{\Gamma_{d}}\left(x_{i}, y_{i}\right)\right) \mathbf{N}_{\mathrm{STD}}^{T} \mathbf{t} d \Gamma+\int_{\Gamma_{d^{-}}}\left(\mathscr{H}_{\Gamma_{d}}(x, y)-\mathscr{H}_{\Gamma_{d}}\left(x_{i}, y_{i}\right)\right) \mathbf{N}_{\mathrm{STD}}{ }^{T}(-\mathbf{t}) d \Gamma \\
& =\int_{\Gamma_{d}} \mathbf{N}_{\mathrm{STD}}^{T} \mathbf{t} d \Gamma
\end{aligned}
$$

Substituting Eq. (15) into Eq. (13) results Eq. (16): 


$$
\left[\begin{array}{ll}
\mathbf{K}^{u u} & \mathbf{K}^{u a} \\
\mathbf{K}^{a u} & \mathbf{K}^{a a}
\end{array}\right]\left[\begin{array}{l}
\mathbf{u} \\
\mathbf{a}
\end{array}\right]=\left[\begin{array}{c}
\mathbf{f}_{u}^{e x t} \\
\int_{\Gamma_{d}} \mathbf{N}_{\mathrm{STD}}{ }^{T}\left(H_{\Gamma_{d}}(x, y)-H_{\Gamma_{d}}\left(x_{i}, y_{i}\right)\right) \overline{\mathbf{t}} d \Gamma
\end{array}\right]+\left[\begin{array}{c}
0 \\
\mathbf{f}_{c o h}
\end{array}\right]
$$

To be able to reach a fully condensed equilibrium system, the additional enrichment term $\mathbf{a}$ is eliminated, thus the equilibrium equation with the standard FEM unknown quantities can be consequently obtained as shown in Eq. (17):

$$
\left(\mathbf{K}^{u u}-\mathbf{K}^{u a}\left(\mathbf{K}^{a a}\right)^{-1} \mathbf{K}^{a u}\right) \mathbf{u}=\mathbf{f}_{\text {ext }}^{u}-\mathbf{K}^{u a}\left(\mathbf{K}^{a a}\right)^{-1} \mathbf{M} \mathbf{f}_{e x t}^{u}-\mathbf{K}^{u a}\left(\mathbf{K}^{a a}\right)^{-1} \mathbf{f}_{c o h}
$$

Where, $\mathbf{M}$ is a transformation matrix to $\operatorname{link} \mathbf{f}_{e x t}^{a}$ and $\mathbf{f}_{e x t}^{u}$ as bellows.

$$
\mathbf{f}_{e x t}^{a}=\mathbf{M f}_{e x t}^{u}
$$

It should be noted that in the most case $\mathbf{M}$ is zero because there is no external force applied on the cohesive crack. Thus Eq. (17) can be rewritten as:

$$
\left(\mathbf{K}^{u u}-\mathbf{K}^{u a}\left(\mathbf{K}^{a a}\right)^{-1} \mathbf{K}^{a u}\right) \mathbf{u}=\mathbf{f}_{e x t}^{u}-\mathbf{K}^{u a}\left(\mathbf{K}^{a a}\right)^{-1} \mathbf{f}_{c o h}
$$

This ECDM formulation is a lower order equilibrium system compared to standard XFEM, and the ECDM permits nodal displacement calculation of the cracked element using standard FEM DoFs only.

In the equilibrium Eq. (19), the sub matrix of elemental stiffness matrix can all be obtained by integration within the $2 \mathrm{D}$ element domain $\Omega_{\mathrm{e}}$. It is assumed that there are no external boundary conditions prescribed on the cracked ECDM element. The submatrices of the elemental stiffness matrix can be given as below:

$$
\begin{aligned}
\mathbf{K}^{u u} & =\int_{\Omega_{e}^{+}} \mathbf{B}_{\mathrm{STD}}{ }^{T} \mathbf{D} \mathbf{B}_{\mathrm{STD}} \mathrm{d} \Omega+\int_{\Omega_{e}^{-}} \mathbf{B}_{\mathrm{STD}}{ }^{T} \mathbf{D} \mathbf{B}_{\mathrm{STD}} d \Omega \\
\mathbf{K}^{a u} & =\int_{\Omega_{e}^{+}} \mathbf{B}_{\mathrm{ENR}}{ }^{T} \mathbf{D} \mathbf{B}_{\mathrm{STD}} d \Omega+\int_{\Omega_{e}^{-}} \mathbf{B}_{\mathrm{ENR}}{ }^{T} \mathbf{D} \mathbf{B}_{\mathrm{STD}} d \Omega \\
\mathbf{K}^{u a} & =\int_{\Omega_{e}^{+}} \mathbf{B}_{\mathrm{STD}}{ }^{T} \mathbf{D} \mathbf{B}_{\mathrm{ENR}} d \Omega+\int_{\Omega_{e}^{-}} \mathbf{B}_{\mathrm{STD}}{ }^{T} \mathbf{D} \mathbf{B}_{\mathrm{ENR}} d \Omega \\
\mathbf{K}^{a a} & =\int_{\Omega_{e}^{+}} \mathbf{B}_{\mathrm{ENR}}{ }^{T} \mathbf{D} \mathbf{B}_{\mathrm{ENR}} d \Omega+\int_{\Omega_{e}^{-}} \mathbf{B}_{\mathrm{ENR}}{ }^{T} \mathbf{D} \mathbf{B}_{\mathrm{ENR}} d \Omega
\end{aligned}
$$

where $\mathbf{D}$ is the constitutive tangent matrix of material. The elemental stiffness matrix Eq. (20) is a $8 \times 8$ matrix, thus there are totally 8 DoFs for the ultimate implemented ECDM element, which is the same with conventional 2D quadrilateral element. No extra DoFs or nodes are needed in the ultimate implementation of the ECDM. In the programming, the above integration 
to construct elemental stiffness matrix is performed within the natural element as shown in Fig. 2(b). Hence the submatrices shown in Eq. (20) can be expressed as Eq. (21) through area mapping:

$$
\begin{aligned}
& \mathbf{K}^{u u}=\int_{\Xi_{e}^{+}} \mathbf{B}_{\mathrm{STD}}{ }^{T} \mathbf{D} \mathbf{B}_{\mathrm{STD}} \operatorname{det}|\mathbf{J}| \mathrm{d} \boldsymbol{\Xi}+\int_{\Xi_{e}^{-}} \mathbf{B}_{\mathrm{STD}}{ }^{T} \mathbf{D} \mathbf{B}_{\mathrm{STD}} \operatorname{det}|\mathbf{J}| d \boldsymbol{\Xi} \\
& \mathbf{K}^{a u}=\int_{\Omega_{e}^{+}} \mathbf{B}_{\mathrm{ENR}}{ }^{T} \mathbf{D} \mathbf{B}_{\mathrm{STD}} \operatorname{det}|\mathbf{J}| d \boldsymbol{\Xi}+\int_{\Omega_{e}^{+}} \mathbf{B}_{\mathrm{ENR}}{ }^{T} \mathbf{D} \mathbf{B}_{\mathrm{STD}} \operatorname{det}|\mathbf{J}| d \boldsymbol{\Xi} \\
& \mathbf{K}^{u a}=\int_{\Xi_{e}^{+}} \mathbf{B}_{\mathrm{STD}}{ }^{T} \mathbf{D} \mathbf{B}_{\mathrm{ENR}} \operatorname{det}|\mathbf{J}| d \boldsymbol{\Xi}+\int_{\Xi_{e}^{+}} \mathbf{B}_{\mathrm{STD}}{ }^{T} \mathbf{D} \mathbf{B}_{\mathrm{ENR}} \operatorname{det}|\mathbf{J}| d \boldsymbol{\Xi} \\
& \mathbf{K}^{a a}=\int_{\Xi_{e}^{+}} \mathbf{B}_{\mathrm{ENR}}{ }^{T} \mathbf{D} \mathbf{B}_{\mathrm{ENR}} \operatorname{det}|\mathbf{J}| d \boldsymbol{\Xi}+\int_{\Xi_{e}^{+}} \mathbf{B}_{\mathrm{ENR}}{ }^{T} \mathbf{D} \mathbf{B}_{\mathrm{ENR}} \operatorname{det}|\mathbf{J}| d \boldsymbol{\Xi}
\end{aligned}
$$

Where, $\boldsymbol{\Xi}_{e}^{+}$and $\boldsymbol{\Xi}_{e}^{-}$are the integration domains (in natural coordinates) corresponding to $\boldsymbol{\Omega}_{e}^{+}$ and $\boldsymbol{\Omega}_{e}^{-}$(in physical coordinates), respectively. By applying different constitutive ma trix of material in Eq. (21), simulating the mechanical behaviors of plane stress and plane strain problems for both isotropic and orthotropic material can be achieved.

\section{Calculation of the nodal force attributing to cohesion}

In a 2D 4-node ECDM element hosting a crack as illustrated in Fig. 3(a), taking account for the existence of cohesive segment, the internal nodal force vector $\mathbf{f}_{\text {coh }}$ due to cohesive traction on the crack surface $\boldsymbol{\Gamma}_{\text {element }}^{\text {crack }}$ can be expressed as:

$$
\mathbf{f}_{c o h}=\int_{\Gamma_{\text {element }}^{\text {crack }}} \mathbf{N}_{\mathrm{STD}}^{T} \mathbf{t} d \Gamma
$$

in which $\mathbf{t}$ is the traction between the two crack surfaces within the specified failure element, as shown in Fig . 3(b). The integration of the internal nodal force vector due to cohesive traction $\mathbf{t}$ shown in Eq. (22) can be completed by numerical integration about the cohesive traction on elemental crack surface $\boldsymbol{\Gamma}_{\text {element }}^{\text {crack }}$. In this work, the standard Gaussian integration scheme is employed, which is expressed as:

$$
\mathbf{f}_{c o h}=\int_{\Gamma_{\text {coch }}^{\text {crack }}} \mathbf{N}_{\mathrm{STD}}^{T} \mathbf{t} d \Gamma=\sum_{i=1}^{n} \mathbf{N}_{\mathrm{STD}}^{T}\left(\xi_{\mathrm{i}}\right) \mathbf{t}\left(\xi_{\mathrm{i}}\right) \mathrm{Z}\left(\xi_{\mathrm{i}}\right)
$$

Where, $\xi_{i}$ is the coordinates of $i_{\text {th }}$ gauss integration point of total $n$ Gauss points, with the $Z\left(\xi_{i}\right)$ weight function. Herein, two gauss points are used for integration, whose coordinates 
are $(-1 / \sqrt{3})$ and $(1 / \sqrt{3})$. Within any specified failure elements, the status of cohesive segment is regarded to be consistent in this implementation of the ECDM.

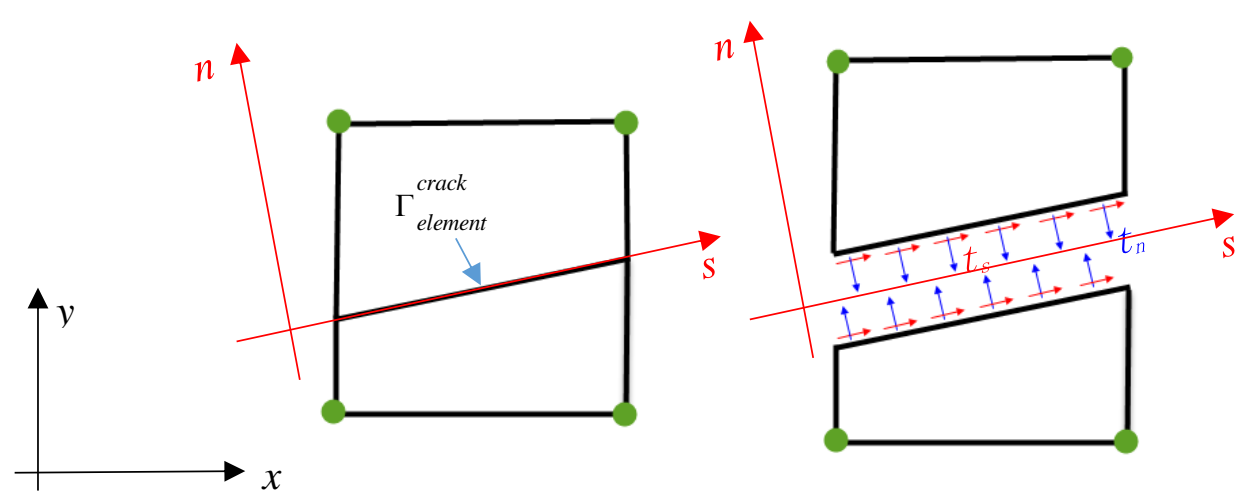

Fig.3. Elemental cohesive traction distribution between the crack surfaces with cohesion.

As it is described in Chapter II, the initial cohesive traction between the cracked surfaces of a failure element can be calculated as the normal stress and shearing stress on the cracked surface. In order to obtain the initial cohesive traction $\mathbf{t}_{0}$, the initial stress status $\boldsymbol{\sigma}_{0}$ at the center point of the crack length within specified element is needed and calculated by the following formula:

$$
\boldsymbol{\sigma}_{\mathbf{0}}=\left[\begin{array}{lll}
\sigma_{0}^{x} & \sigma_{0}^{y} & \tau_{0}^{x y}
\end{array}\right]=\mathbf{D B} \mathbf{u}_{\mathbf{0}}
$$

in which $\mathbf{u}_{0}$ is the nodal displacement when the crack propagates. Subsequently, the calculation of initial cohesive traction $\mathbf{t}_{0}$ in the local $(s, n)$ coordinate whose $s$-axis is aligned along the crack direction (angle between coordinates $(s, n)$ and $(x, y)$ is $\alpha$ ) can be carried via:

$\mathbf{t}_{0}=\left[\begin{array}{c}t_{0}^{n} \\ t_{0}^{s}\end{array}\right]=\left[\begin{array}{c}\langle\sigma\rangle_{0}^{n} \\ \sigma_{0}^{s}\end{array}\right]=\left[\begin{array}{c}\left\langle\frac{\sigma_{0}^{x}+\sigma_{0}^{y}}{2}+\frac{\sigma_{0}^{x}-\sigma_{0}^{y}}{2} \cos 2 \alpha-\tau_{0}^{x y} \sin 2 \alpha\right\rangle \\ \frac{\sigma_{0}^{x}-\sigma_{0}^{y}}{2} \sin 2 \alpha+\tau_{0}^{x y} \cos 2 \alpha\end{array}\right]$

in above equation, $<>$ is the Ma-Cauley bracket operator that $\langle\mathrm{A}\rangle$ designates the value of $\langle A\rangle=\frac{A+|A|}{2}$, with the introduction of which, the longitudinal strain energy density can be distinguished between the tension behavior and the compression behavior.

\section{Verification of the ECDM via single element tests}


A displacement-controlled loading procedure is applied on a single element to test the performance of the ECDM in capturing fracture behavior. Different loading conditions, which can lead to different fracture modes, i.e. mode I, II and mixed mode fracture, will be applied. The dimension for both length and height of the tested element is $l=5 \mathrm{~mm}$. The potential discontinuity in the element is laid from center of left edge to the center of right edge. The linear softening cohesive law is selected to test through this modeling simulation. The material properties used for single element tests are randomly selected as: Young's modules $E=1000 \mathrm{Mpa}$, Poison' ratio $v=0.3$, normal tensile strength $\sigma_{n}=25 \mathrm{MPa}$, shear strength $\sigma_{S}=25 \mathrm{MPa}$, fracture energy $G c=0.1 \mathrm{~N} / \mathrm{mm}$.

For the purpose of assessing the performance of the ECDM, i.e. the accuracy in the predicting both the peak loading value and the post-peak response, the modelling with the standard $2 \mathrm{D}$ elements in ABAQUS is also carried out and the results will be compared with the ECDM solutions. Regular plane stress elements (CPS4) with elastic material behavior are used for the modelling of top and bottom subdomains, and cohesive elements (COH2D4) with zero thickness are inserted between the two CPS4 elements to account for the fracture nonlinearity. The same geometry and material properties are used for both the ECDM and standard ABAQUS modelling.

\subsection{Mode I response}

For the mode I fracture test, nodes 1 and 2 are fixed while nodes 3 and 4 are subjected to vertical displacement. In doing so, the developed fracture will be only the opening mode-mode I. In Fig. 4, the nodal force versus applied displacement response is plotted, from which it can been seen that the total peak nodal forces at nodes 3 and 4 are $1.0 \mathrm{~N} / \mathrm{mm}$ and the element is completely cracked at the critical displacement of $0.1 \mathrm{~mm}$. Identical result curve are obtained by the modelling with ABAQUS cohesive element. 

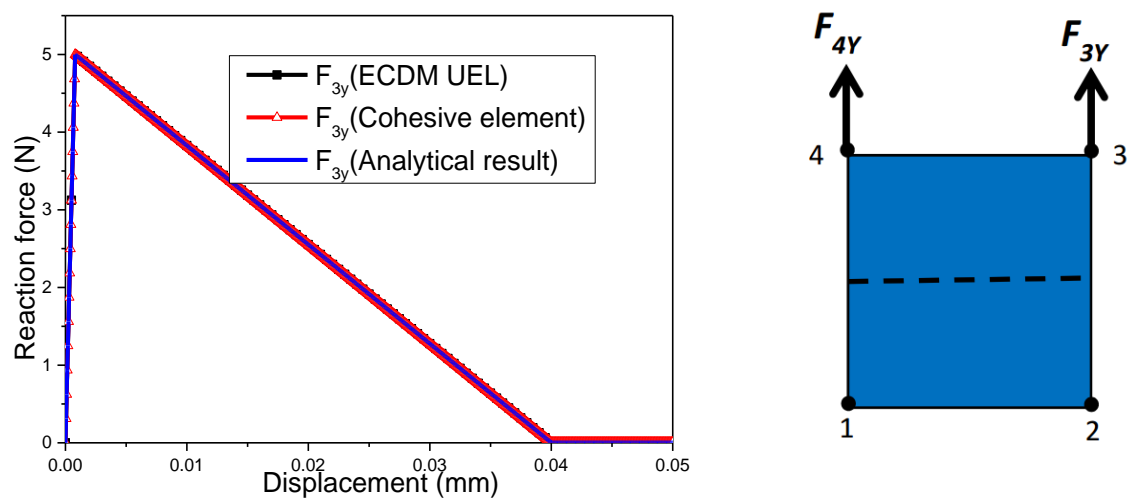

Fig. 4. A single ECDM element response under mode I loading

In the single mode fracture cases, the analytical peak force is available. For this problem, the peak value can be calculated by the $F_{3 y}=F_{4 y}=l \sigma_{22} / 2$, which is consistent with the ECDM solution. It should be noted that $\sigma_{22}$ is the tension stress calculated by the ECDM. Comparison of the deformed shapes from the ECDM and the standard ABAQUS cohesive modelling is shown in Fig. 5. Both the ECDM and the standard ABAQUS cohesive element predict a same deformation of the single element under tension.

Based on the above comparison, it can be confirmed that the modelling of the above Mode I fracture process can be completed by using only one ECDM element to achieve the same accuracy as that obtained by the ABAQUS cohesive modeling consisting of three elements as shown in Fig. 5(b), two plane stress elements for elastic material behavior and one cohesive element for nonlinear fracture.

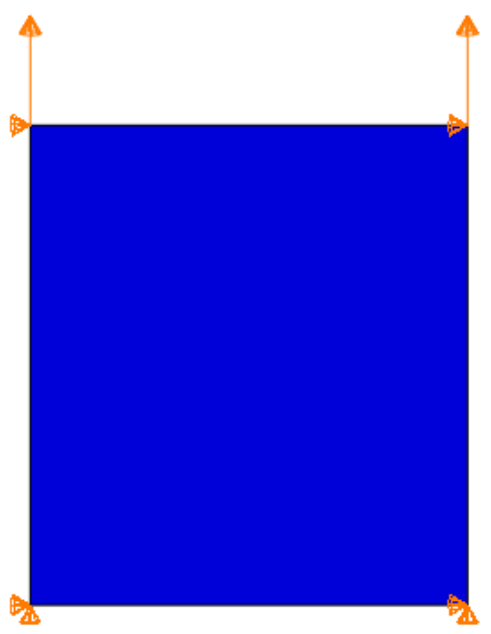

(a)

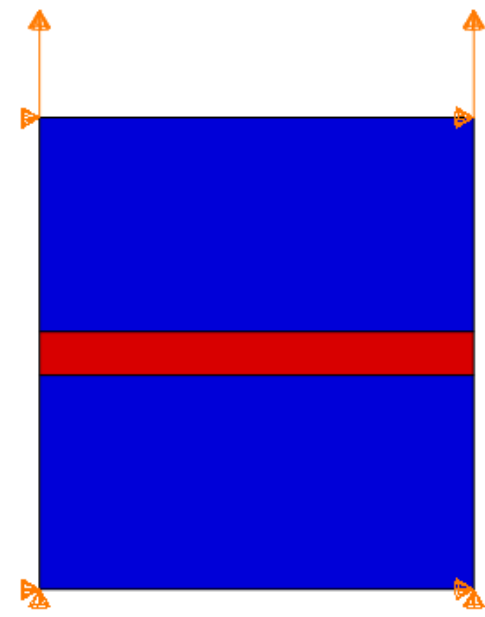

(b) 
Fig. 5. Mode I deformation pattern predicted by (a) the ECDM user element and (b) ABAQUS cohesive element.

\subsection{Mode II response}

To assess the performance of the ECDM user element in modelling of Mode II fracture, horizontal displacements are applied on node 3 and node 4 at the same single element used in mode I fracture test. Fig. 6 shows a good agreement in failure response between the numerical results and analytical result. Fig. 7 shows the comparison of the response between the ECDM predictions and the results from cohesive element. Pure shear deformation can be observed in the element. The predictions of nodal reaction forces from both the ECDM and cohesive element are identical.
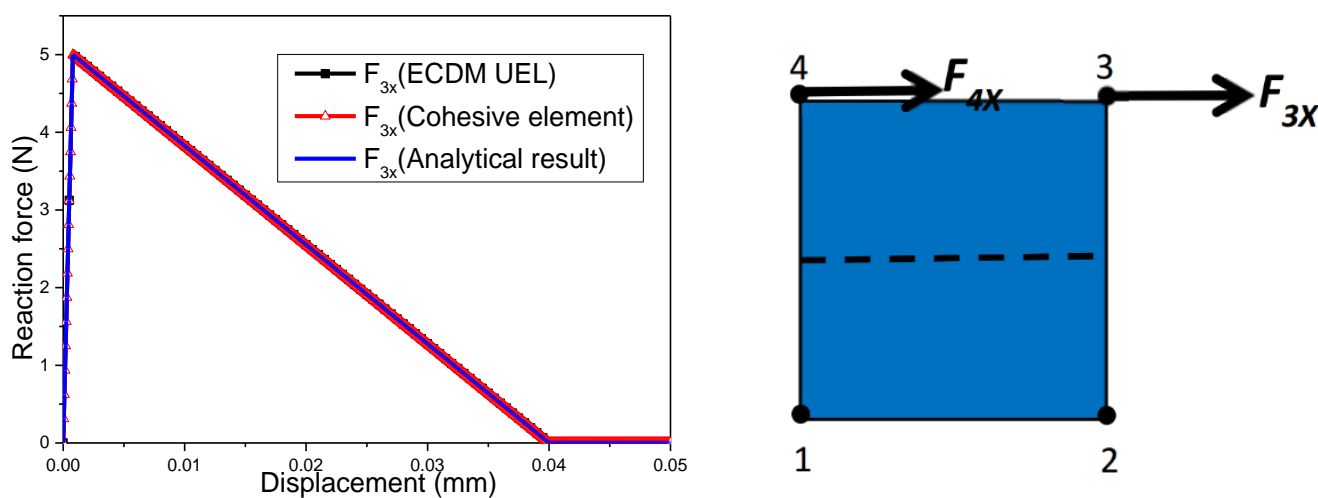

Fig. 6. A single ECDM element response under mode II loading

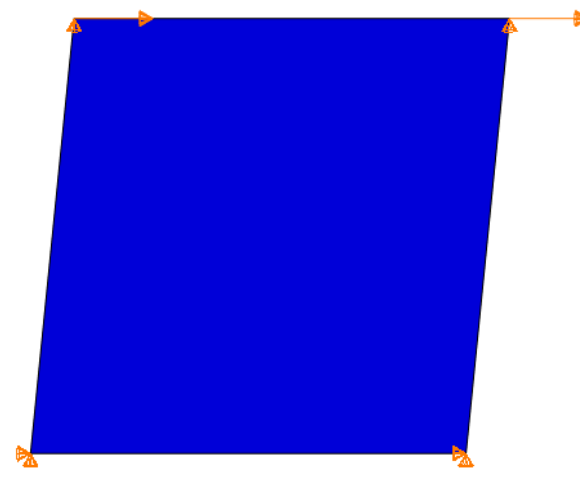

(a)

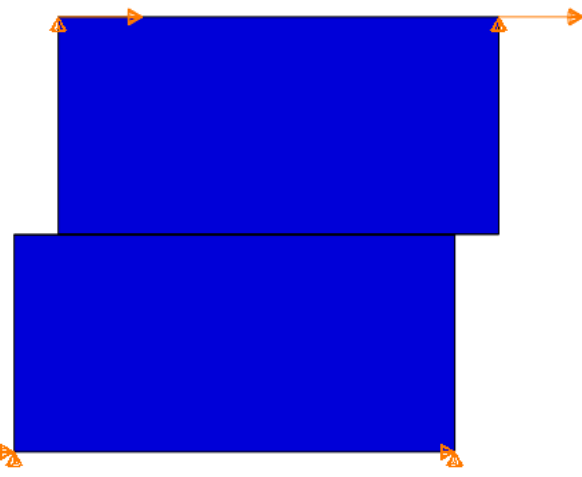

(b)

Fig. 7. Mode-II deformation predicted by (a) the ECDM user element and (b) ABAQUS cohesive element 


\subsection{Mixed Mode response}

A mixed mode loading scenario will be performed via combining the mode I and mode II loading conditions together to test the performance of the ECDM user element in modeling the mix-mode fracture. The comparison of the nodal reaction between the ECDM predictions and the results from cohesive element modelling is presented in Fig. 8, which shows a very good agreement. The deformation due to mixed mode fracture is presented in Fig. 9. It can be seen from Fig. 9 that the ECDM can reproduce the nodal displacement accurately but not the physical displacement jump which can be predicted by cohesive element modelling. However, the ECDM can alternatively express the discontinuity using the maximum principal strain contour. This can be seen from the ECDM based structural models e.g. a specimen with delamination migration given in the next section.
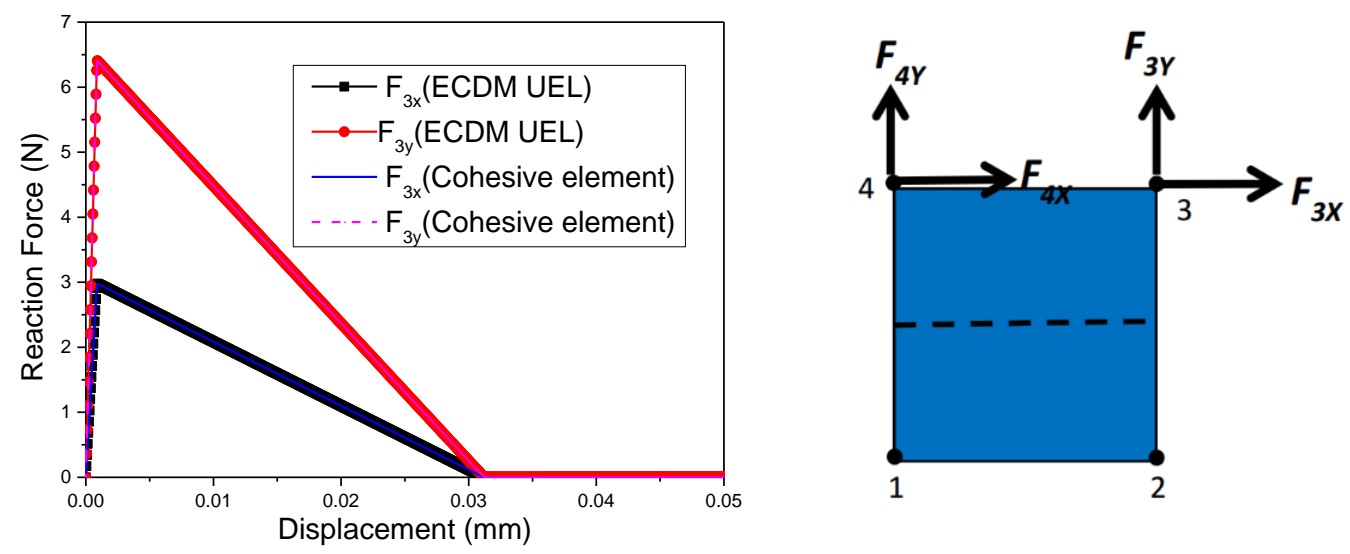

Fig. 8. A single ECDM element response under mixed mode loading

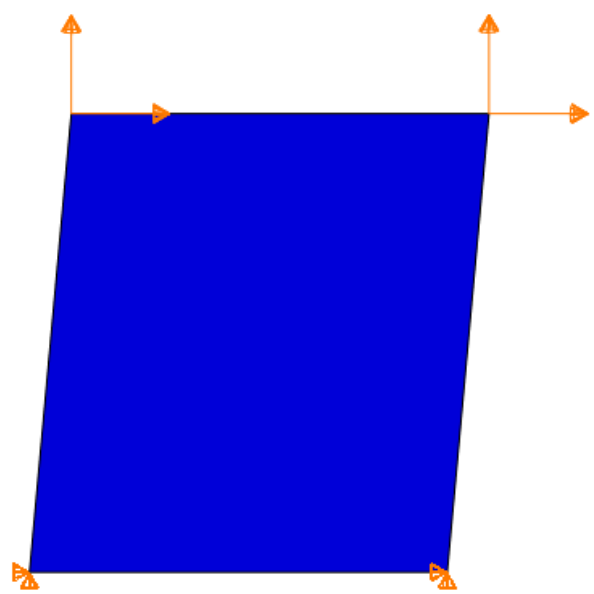

(a)

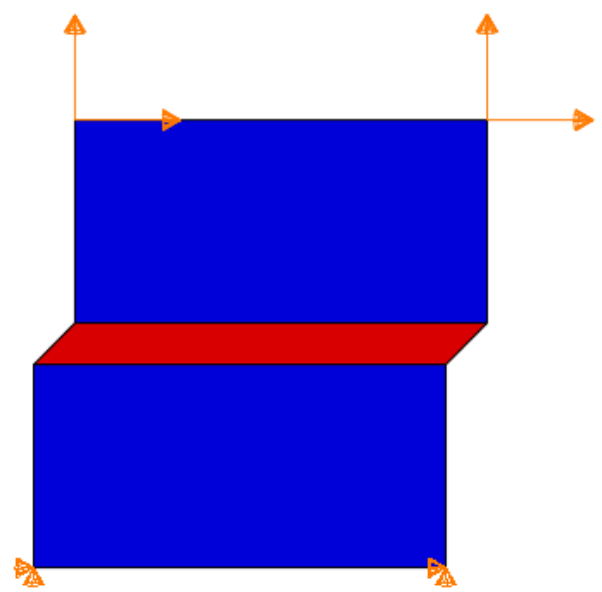

(b) 
Fig. 9. Mixed mode deformation pattern predicted by (a) the ECDM user element and (b) cohesive element

\section{Prediction of delamination migration in laminated composites}

In engineering, loading condition applied on FRP structure is always complex, subjected to which multiple failure modes may be provoked. These multiple failure modes may develop synchronously and independently. The coupling effect between different failure modes is normally critical. Hence, a profound knowledge of the failure mechanisms of FRP composites is essentially required for efficiently and economically instructing the practical design of FRP composite structures. Based on this consideration, the modelling of the multiple failure mechanisms is essential to the in-depth investigation of failure mechanisms in FRP composites. Laminated composite structure is typical FRP composites, whose failure mechanisms and multiple failure modes are complex. Considering multiple damage behaviors, delamination is usually found when structures loose loading capacity. Therefore, it is critical to investigate the delamination mechanism in the design of laminated composites.

This developed ECDM featuring with arbitrary crack propagation and elimination of enriched degree freedoms is believed to be suitable for the modelling of delamination migration. Some initial applications of the ECDM in modelling fracture mechanisms in FRP composites can been found in $[23,24]$. Current existing numerical techniques such as XFEM in FEM package of ABAQUS hardly deal with the problem of multiple failure mechanism in FRP composite laminates. By implementing different failure criterion and damage evolution schemes, the ECDM can overcome such drawback in XFEM and therefore can predict complex fracture behavior, e.g. delamination migration $[18,19]$, in which not only delamination but also matrix cracking can be simulated.

\subsection{A multicrack propagation scheme in fibre laminated composites}

Multiple fracture modes including matrix crack, fibre breakage and delamination in fibre laminated composites are schematically illustrated in Fig. 10. In the case of matrix crack and fiber fracture, a maximum principal stress based criteria is used to characterize the damage 
initiation, which means when the maximum principal stress $\sigma_{\max }$ at gauss points of any elements is beyond the cohesive strength $\mathrm{S}_{\text {matrix }}$ for matrix or $\mathrm{S}_{\text {fibre }}$ for fibre, the damage onsets. The perpendicular direction to the direction of the maximum principal stress is adopted to be the crack direction within the cracked element, which provides a potential arbitrary crack propagation. It should be noted that a brittle fracture is assumed in the case of fibre breakage. As shown in Fig. 10, the average maximum principal stresses from four Gauss points at the upper layer $\left(90^{\circ} \mathrm{ply}\right)$ and the lower layer $\left(0^{\circ} \mathrm{ply}\right)$ are calculated for the judgment of matrix crack and fiber breakage, respectively. The fracture directions of matrix crack and fiber breakage are veraciously [18] determined by the $\theta_{\max }$, which is perpendicular to the direction of the maximum principal stress. On the presence of delamination, a stress based criteria given in the Fig. 10 and the stresses of the mid-point at the interface between two plies are used for the judgment of damage initiation. Considering a general mix-mode delamination propagation, a mix-mode criterion proposed by Benzeggagh and Kenane [20], i.e. a total fracture energy as a function of crack mode ratio given in Eq. (26) is used in this investigation.

$$
G_{f}=G_{f, I}+\left(G_{f, I I}-G_{f, I}\right)\left(\frac{1}{1+\beta}\right)^{\eta}
$$

Where the mix-mode parameter $\eta$ is taken as $1.39[10] . G_{f, I}$ and $G_{f, I I}$ are fracture energy corresponding to pure mode I and mode II fracture, respectively. For a normal opening delamination case, the mix-mode ration $\beta$ is defined as:

$$
\beta=\left|\frac{\sigma_{n}^{0} / N}{\sigma_{s}^{0} / s}\right|
$$

Where $\sigma_{n}^{0}$ and $\sigma_{s}^{0}$ are the normal and shear initial stress, respectively, corresponding to the onset of damage initiation under mix-mode loading; $N$ and $S$ are the material strength corresponding to Mode I and Mode II fracture, respectively. The path of delamination propagation is prescribed along the physical interface boundary when $\theta_{\max }$ is zero.

\subsection{Configuration of the specimen with delamination migration}


Fig. 11 shows the basic configuration of the investigated specimen which consists of a crossply IM7/8552 tape laminate with a polytetrafluoroethylene insert implanted at the mid-plane and spanning part way along the length of the specimen. The detailed configuration of the specimen and the test set-up can be referred to [18]. There is an existing delamination with the length of $51 \mathrm{~mm}$ at the $0 / 90$ interface. The lay-up sequence of the laminate is $\left[90_{4} / 0_{3} /(90 / 0)_{2 s} / 0_{3} / 90_{4} / \mathrm{T} / 0 / 90_{4} / 0 / 0 /(90 / 0)_{2 s} / 0 / 0 / 90_{3} / 0 / 90\right]$. The material properties of IM7/8552 lamina are $E_{11}=161.0 \mathrm{GPa}, E_{22}=E_{33}=11.38 \mathrm{GPa}, v_{12}=v_{13}=0.32 \quad v_{23}=0.43 \quad G_{12}=G_{13}=5.17 \mathrm{GPa}$ and $G_{23}=3.98 \mathrm{GPa}$, respectively. Regarding the interfacial fracture parameters, both normal and shear strength are 15.0MPa; fracture energy for both mode I and mode II are $0.25 \mathrm{~N} / \mathrm{mm}$ and $0.65 \mathrm{~N} / \mathrm{mm}$, respectively. To be identical to the test, vertical displacement is conducted at the load-application point located at the front of existing delamination in order to initiate delamination onset and propagation. The delamination continues from the existing delamination along the 0/90 interface then followed by a matric crack as a kinking through the 90-degree ply stack and the delamination migrated to a neighboring 90/0 ply interface.

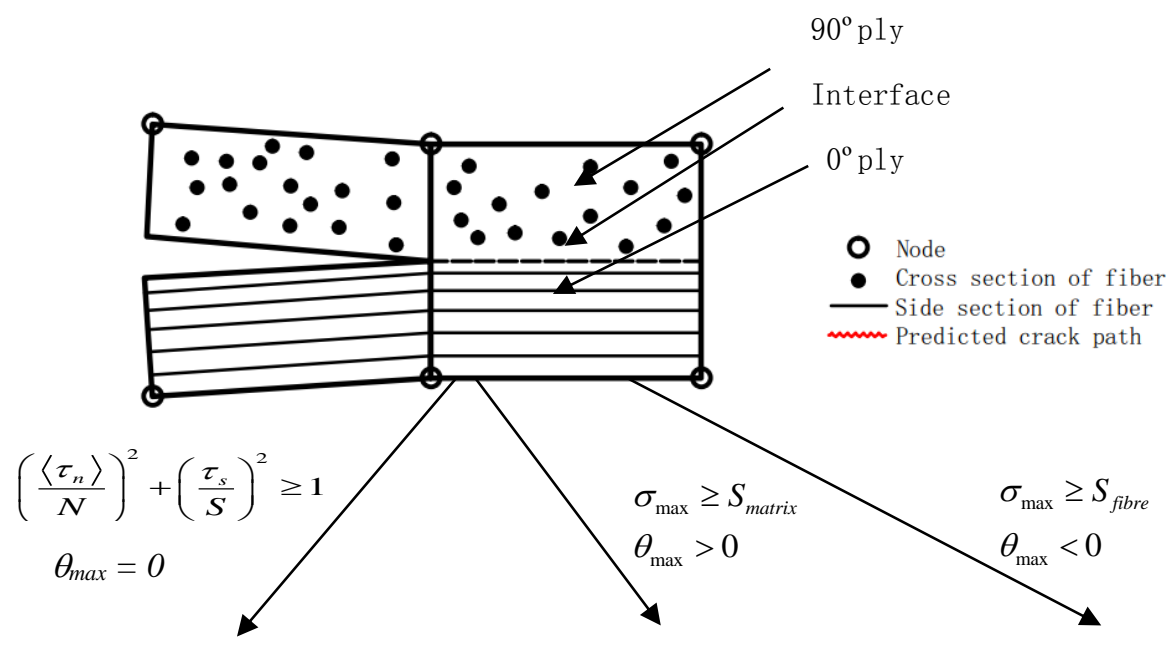

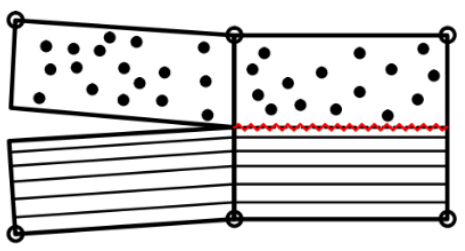

(a) Delamination

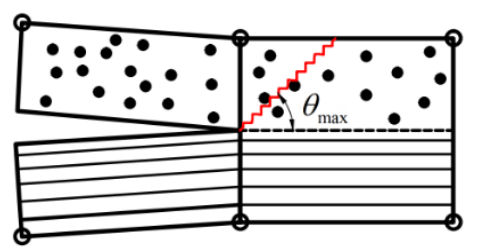

(b) Matrix crack

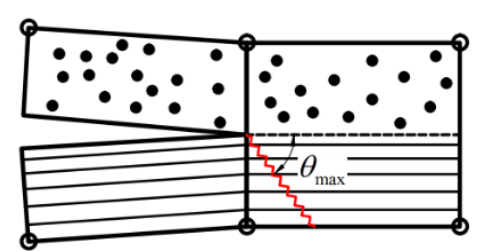

(c) Fibre breakage

Fig. 10. A schematic illustration of failure mechanism judgments in fibre composites. 
For comparison reason, both the ECDM and CZM are used in this modelling analysis. The FE mesh configurations of the highlighted region by dash line in Fig. 11 are presented below, from which the delamination migration was observed from experimental work. It can be seen from Fig. 11 that the mesh configuration using ECDM is uniform, while that for the model using CZM based cohesive element is discretized in conforming the anticipated migration path, and zero thickness cohesive elements are embedded in the mesh for CZM. In CZM modelling, the fracture can only propagate through the path setting a prior. In experimental work [18], different specimens in terms of different load offset, $L$, normalized by the initial delamination length, $\mathrm{a}_{0}$, were tested. In [21] and [22], the behavior of delamination migration has been investigated using floating node method and XFEM, respectively. Herein, the cases with $L=a_{0}=49 \mathrm{~mm}$ and $L=1.2 a_{0}=58.8 \mathrm{~mm}$ are investigated in this paper for the purpose of assessing the performance of ECDM in a highly efficient prediction of delamination migration in laminated composites.

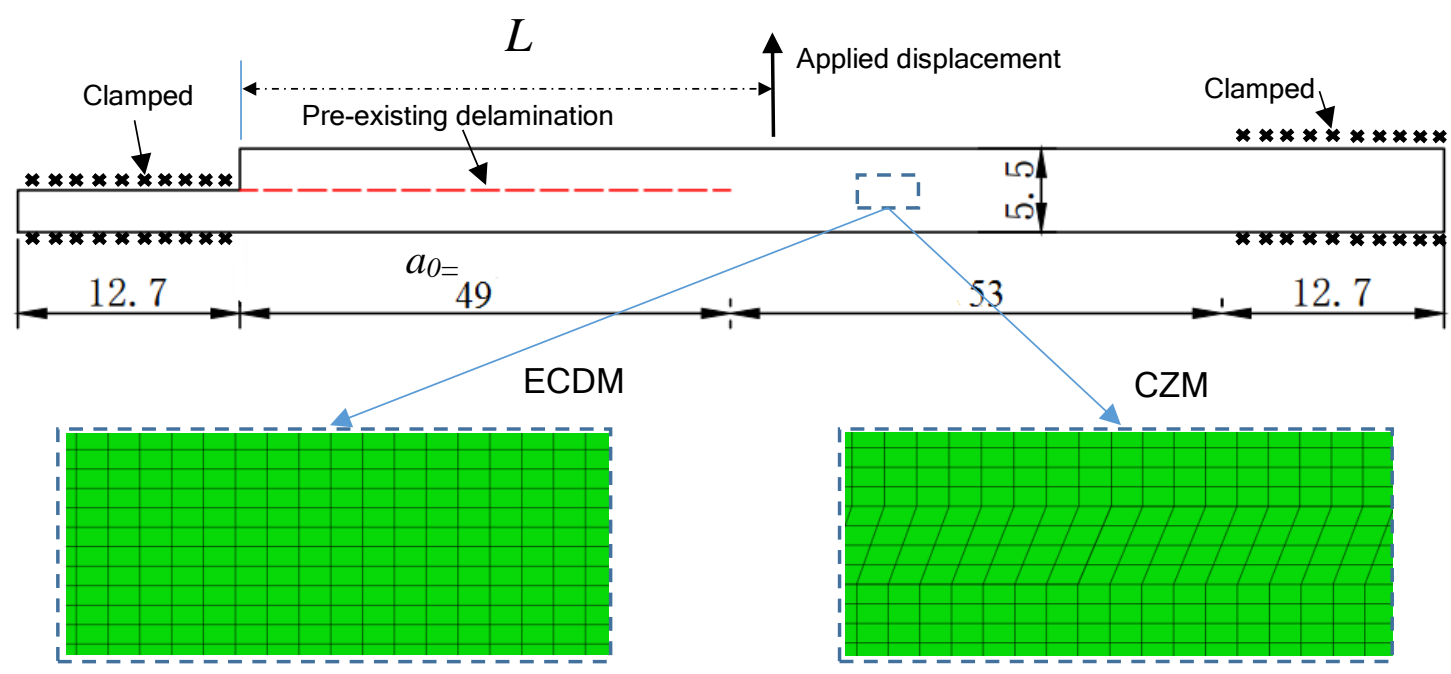

Fig. 11. Configuration of the specimens for delamination migration test and the FE meshes of the highlighted region for the ECDM and CZM (Unit: $\mathrm{mm}$ ).

\subsection{Result analysis}

Fig. 12 shows the ECDM simulated a final failure stage with delamination growth, matrix crack and delamination migration in the case of $L=a_{0}=49 \mathrm{~mm}$. An image of delamination migration from experimental measurement is also given in Fig. 12. The global failure response of this investigated specimen is shown in Fig.13, from which it can be seen that the entire failure response obtained from the modelling is sufficiently consistent with experimental measurement. 
The failure response of the specimen experiences two main sudden drops during the loading process, which represent two major fracture events within the specimen. The first one is the existing delamination growth, the second is matrix crack together with a new delamination propagation, named as delamination migration. When the specimen is loaded over $200 \mathrm{~N}$, the existing delamination propagates approximately $8.6 \mathrm{~mm}$ along the $0^{\circ} / 90^{\circ}$ intralaminar interface, which agrees to the experimental observation between $8 \mathrm{~mm}$ and $9 \mathrm{~mm}$. This delamination is an unstable delamination propagation at the $0^{\circ} / 90^{\circ}$ interface. When the specimen is reloaded up between $150 \mathrm{~N}$ and $180 \mathrm{~N}$ in experiment and around $190 \mathrm{~N}$ by the ECDM, the matrix crack starts and quickly goes through the 90-degree ply along a short slope line from the bottom to top of the $90^{\circ}$ ply. Then it is promptly followed by another delamination as a stable crack propagation at the $90^{\circ} / 0^{\circ}$ interlaminar interface. The second sudden drop in failure response reflects matrix crack and the second delamination propagation which are almost appeared at the same time. The rest of failure response is the residual stiffness of the specimen.

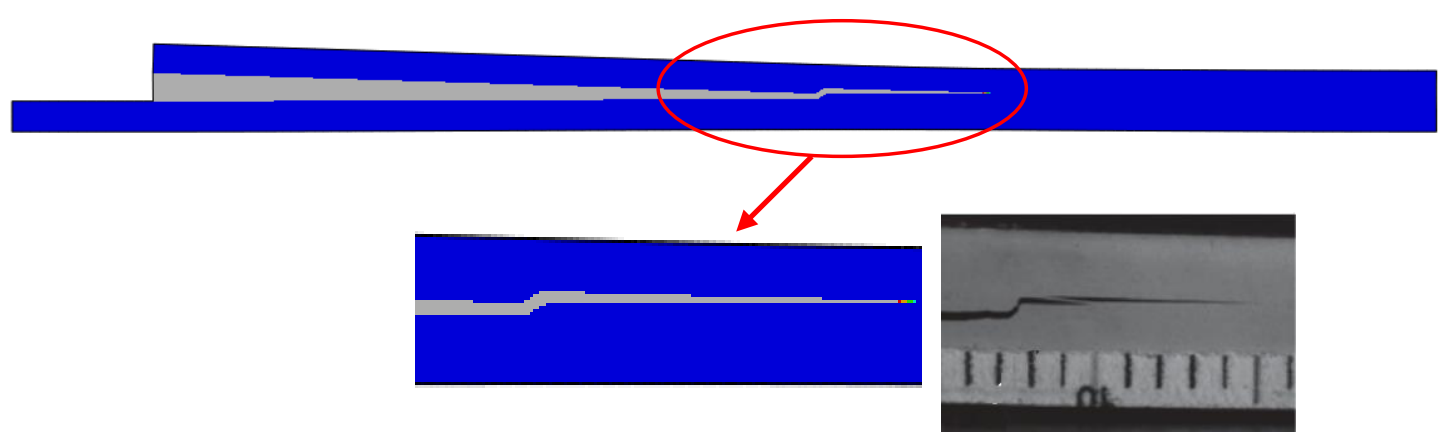

Fig. 12. The ECDM simulated delamination migration and a tested image in the case of $L=a_{0}=49 \mathrm{~mm}$.

This multicrack propagation actually presents the delamination migration through a matrix crack in the investigated specimen. Prior to and after delamination migration the fracture is recognized as mixed mode-I/II crack due to the asymmetrical load condition and specimen configuration. Inspection of the kinked surface in $90^{\circ}$ ply of tested specimen reveals the modeI fracture [18], which proves the modelling prediction of the opening dominated matrix crack. It should be noted that the transition of the kinked crack from $0^{\circ} / 90^{\circ}$ interface into the $90^{\circ} / 0^{\circ}$ interface is sudden, its response together with the second delamination is reflected by the second 
drop in the predicted load-displacement curve and other experimental observation on different samples [18]. This investigation confirmed that the capability of the ECDM in capturing the entire delamination migration path including both interlaminar fracture and intralaminar crack propagation in the investigated laminated composite specimen.

To compare with CZM in modelling delamination migration, the cohesive element COH2D4 in ABAQUS is used in this investigation. An exactly same mesh size with that in the ECDM modelling is used in CZM modelling. The CZM predicted failure response is also presented in Fig. 13 for comparison. It can be seen that the CZM obviously overestimates the first failure load compared to experimental measurement and the ECDM prediction. Besides the cumbersome meshing work in CZM modelling, the CPU time spent by CZM modelling is 16166s, and total increment number is 3344 . In contrast, the CPU time and total increments spent by the ECDM are 1333s and 283, respectively. Both CPU time and total increment number spent by the ECDM are reduced more than $90 \%$ compared to CZM. This comparison certainly shows that the computational efficiency and accuracy of the ECDM is significantly improved compared to CZM.

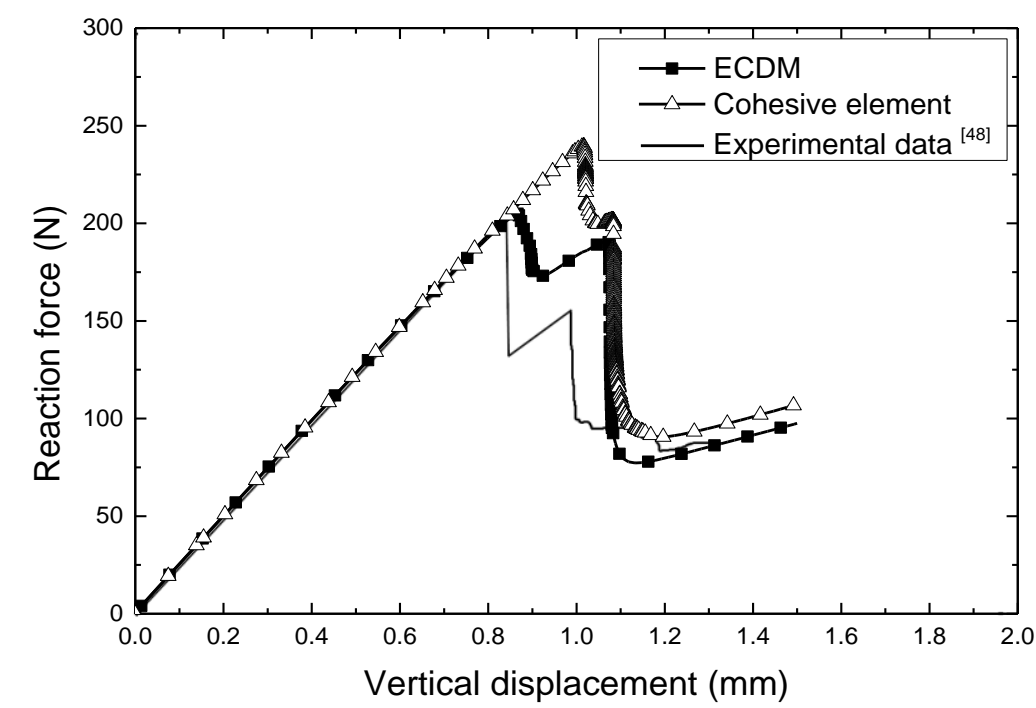

Fig. 13. The load-displacement curves given by the ECDM, CZM and experiment in the case of $L=a_{0}=49 \mathrm{~mm}$.

For the load case of $L=1.2 \mathrm{a}_{0}=58.8 \mathrm{~mm}$, specimen exhibits the same sequence of fracture events as observed in the specimen with $L=\mathrm{a}_{0}=49 \mathrm{~mm}$. The ultimate strength of structure reasonably 
agrees with experimental data, as shown in Fig. 14. Similar to the above discussed failure response, the first drop in load-displacement curve indicates the propagation of an unstable delamination between $0^{\circ} / 90^{\circ}$ layers when load is about $325 \mathrm{~N}$. This unstable delamination stops just before the onset of delamination migration when load drops to $245 \mathrm{~N}$. This predicted step load after the unstable delamination is slightly lower than the experimental observation, which can possibly be attributed to the fiber bridging in experiment (see Fig. 15), which has, in fact, enhanced the specimen. Fig. 15 shows that the ECDM predicted delamination migration is very similar to tested image of the specimen. The overall failure morphology predicted by the ECDM is illustrated in Fig. 15 and compared to the failure morphology observed experimentally. It is predicted that the existing delamination propagates about $8.16 \mathrm{~mm}$ before migration, which is within the experimental envelop observed between 7 and $10 \mathrm{~mm}$.

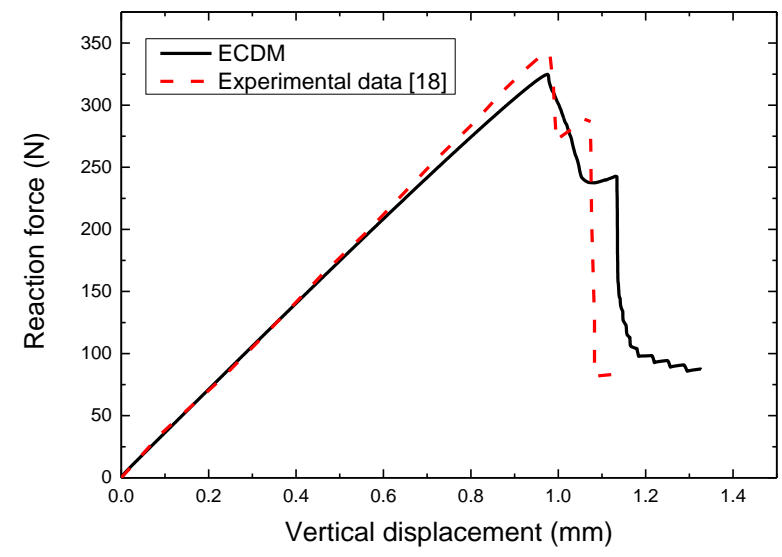

Fig. 14. The load-displacement curves given by the ECDM and experiment in the case of $L=1.2 a_{0}=58.8 \mathrm{~mm}$.
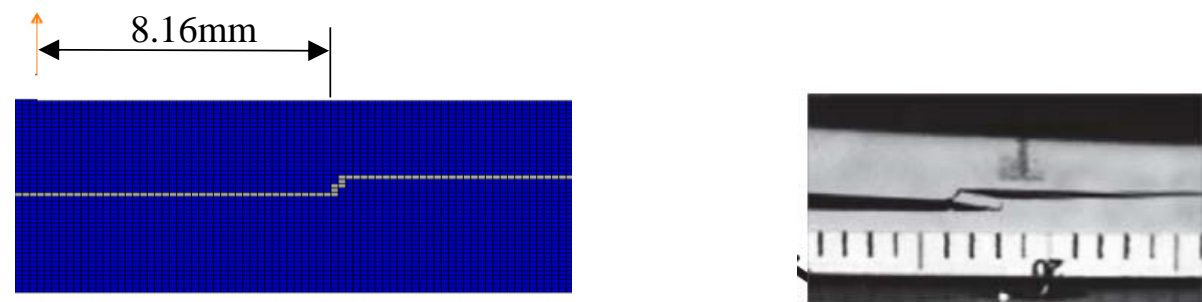

Fig. 15. The ECDM simulated delamination migration and a tested image in the case of $L=1.2 a_{0}=58.8 \mathrm{~mm}$.

\section{Conclusion and future work}


The extended cohesive damage model (ECDM) is developed in this paper, which is based on the framework of XFEM. The enriched degree freedoms required by the standard XFEM are eliminated from the fully condensed equilibrium equations of the ECDM. However, the enrichment effect is reflected by an equivalent stiffness matrix. The cohesive traction is placed into the right hand side of equilibrium equation as part of nodal force vector. The ECDM formulation is verified by a single element modelling in simulating three facture cases with comparison of theoretical calculations. This developed ECDM is successfully used to simulate delamination migration in laminated composites. An example shows the ECDM based modelling can save CPU time by more than $90 \%$ in the modelling of delamination migration compared to CZM. This investigation shows the ECDM is a highly efficient approach in studying fundamental failure mechanism in fibre composites and in predicting multicrack propagation to support the design of composite structures.

The future work includes further application of the ECDM in predicting mulicrack mechanism in different composites such as composite sandwich panels, investigating functionally graded sandwich composites, studying fundamental micro crack mechanism in fibre composites, and further development of the ECDM for predicting failure response of composites subjected to impact load, fatigue load and thermal load. 


\section{Reference}

[1] Hughes, T. J., The finite element method: linear static and dynamic finite element analysis. Courier Corporation 2012.

[2] Oliver, J., \& Huespe, A. E., Theoretical and computational issues in modelling material failure in strong discontinuity scenarios. Computer Methods in Applied Mechanics and Engineering 2004; 193(27): pp.2987-3014.

[3] Chandrasekaran, S., Sato, N., Tölle, F., Mülhaupt, R., Fiedler, B. and Schulte, K., Fracture toughness and failure mechanism of graphene based epoxy composites. Composites Science and Technology 2014; 97: 90-99.

[4] Krueger, R., Cvitkovich, M.K., O'Brien, T.K. and Minguet, P. J., Testing and analysis of composite skin/stringer debonding under multi-axial loading. Journal of composite Materials 2000; 34(15): 1263-1300.

[5] Xiao, Y. and Ishikawa, T., Bearing strength and failure behavior of bolted composite joints (part I: Experimental investigation). Composites Science and Technology 2005; 65(7): 1022-1031.

[6] Maimí, P., Camanho, P.P., Mayugo, J.A. and Dávila, C. G., A continuum damage model for composite laminates: Part I-Constitutive model. Mechanics of Materials 2007; 39(10): 897-908.

[7] Rybicki, E.F. and Kanninen,M.F. (1977). A Finite Element Calculation of Stress Intensity Factors by a Modified Crack Closure Integral, Engineering Fracture Mechanics 1977; 9: 931-938.

[8] Rinderknecht, S. and Kro"plin, B., Calculation of Delamination Growth With Fracture and Damage Mechanics, Recent Developments in Finite Element Analysis, CIMNE 1994; Barcelona, Spain.

[9] Elices, M., Guinea, G.V., Gomez, J. and Planas, J. (2002). The cohesive zone model: advantages, limitations and challenges. Engineering fracture mechanics 2002: 69(2): 137163.

[10] Camanho, P.P., Davila, C.G. and De Moura, M. F., Numerical simulation of mixed-mode progressive delamination in composite materials. Journal of composite materials 2003; 37(16): 1415-1438.

[11] Babuška, I., Melenk, J. M., The partition of unity method, International Journal for Numerical Methods in Engineering 1997; 40: 727-758.

[12] Duarte, C. A., Hamzeh, O. N., Liszka, T. J., \& Tworzydlo, W. W., A generalized finite element method for the simulation of three-dimensional dynamic crack propagation. Computer Methods in Applied Mechanics and Engineering 2001; 190(15): 2227-2262.

[13] Oden, J. T., Duarte, C. A. M., \& Zienkiewicz, O. C., (1998). A new cloud-based hp finite element method. Computer methods in applied mechanics and engineering 1998; 153(1): 117-126. 
[14] Belytschko, T., \& Black, T., Elastic crack growth in finite elements with minimal remeshing. International Journal for Numerical Methods in Engineering 1999; 45: 601620.

[15] Chessa, J., Smolinski, P., \& Belytschko, T., The extended finite element method (XFEM) for solidification problems. International Journal for Numerical Methods in Engineering 2002; 53(8): 1959-1977.

[16] Giner, E., Sukumar, N., Tarancon, J. E., \& Fuenmayor, F. J., An Abaqus implementation of the extended finite element method. Engineering fracture mechanics 2009; 76(3): 347368.

[17] Gigliotti, L., Assessment of the applicability of XFEM in Abaqus for modeling crack growth in rubber, 2012.

[18] Ratcliffe, J.G., Czabaj, M.W. and O'Brien, T. K., A test for characterizing delamination migration in carbon/epoxy tape laminates, 2013.

[19] Pernice, M.F., De Carvalho, N.V., Ratcliffe, J.G. and Hallett, S. R., Experimental study on delamination migration in composite laminates.Composites Part A: Applied Science and Manufacturing, 2015; 73: 20-34.

[20] Benzeggagh, M. L., \& Kenane, M., Measurement of mixed-mode delamination fracture toughness of unidirectional glass/epoxy composites with mixed-mode bending apparatus. Composites science and technology 1996; 56(4): 439-449.

[21] De Carvalho, N.V., Chen, B.Y., Pinho, S.T., Ratcliffe, J.G., Baiz, P.M. and Tay, T. E., Modeling delamination migration in cross-ply tape laminates.Composites Part A: Applied Science and Manufacturing 2015; 71: 192-203.

[22] Zhao, L., Zhi, J., Zhang, J., Liu, Z. and Hu, N., XFEM simulation of delamination in composite laminates. Composites Part A: Applied Science and Manufacturing 2016; 80: $61-71$

[23] X Li and J Chen, An extended cohesive damage model for simulating multicrack propagation in fibre composites, Composite Structures 2016; 143: 1-8.

[24] X Li and $\mathrm{J}$ Chen, The implementation of the extended cohesive damage model for multicrack evolution in laminated composites. Composite Structures, 2016: 139: 68-76. 